PROCEEDINGS OF THE

AMERICAN MATHEMATICAL SOCIETY

Volume 125, Number 12, December 1997, Pages 3725-3733

S 0002-9939(97)04096-3

\title{
OPEN MAPPINGS INCREASING ORDER
}

\author{
JANUSZ J. CHARATONIK AND WŁODZIMIERZ J. CHARATONIK
}

(Communicated by Franklin D. Tall)

\begin{abstract}
It is shown that an analog of Whyburn's theorem saying that open mappings do not increase order of a point of locally compact metric spaces is not true if the Menger-Urysohn order is replaced by order in the classical sense. On the other hand, this analog is true, even for a wider class of confluent mappings, under an additional condition that the mapping is light and the domain continuum is hereditarily unicoherent.
\end{abstract}

\section{INTRODUCTION}

One of the important concepts related to the structure of curves is that of an order of a point in a curve (or, more generally, in a continuum or even in an arbitrary space). As early as in 1906, W. H. Young and G. Ch. Young considered this concept in their book ([13, p. 219-221]) for planar curves in the sense of Cantor (that is, for closed connected subsets of the plane having empty interior). Namely, Young and Young defined a point $p$ in a curve $X$ to be of order $k$ provided there are in $X$ exactly $k$ continua, every two of which have $p$ as the only common point. A further study of this notion is made by Z. Janiszewski in his thesis [5]. Trying to give a more precise definition he used irreducible continua in place of arbitrary ones. Modifying the Young's definition of a point of order $k$ in a continuum, Janiszewski assumed (see $[5$, Chapter $4, \S 1]$ ) that the continua under consideration (which are mutually disjoint out of the point $p$ ) have to be irreducible. He also additionally assumed that their union forms a neighborhood of $p$ (he calls such points regular ones), but the condition is not satisfied at each point of a continuum. To see this, consider, e.g., the union $U$ of countably many circles $C_{n}$ with $\lim \operatorname{diam} C_{n}=0$ and such that $C_{n} \cap C_{n+1}$ is a singleton $\left\{p_{n}\right\}$; if $p=\lim p_{n}$, then $U \cup\{p\}$ is a continuum, and no irreducible continuum (i.e. an arc) containing $p$ form a neighborhood of $p$. Thus, if the notion has to be applicable to any point of an arbitrary continuum, we cannot accept this additional condition. Note however, that the Janiszewski-Mazurkiewicz theorem on the existence of an irreducible continuum in any continuum which contains two given points (see [7, §48, I, Theorem 1, p. 192]; compare [12, Chapter 1, (11.2), p. 17]) implies that these two concepts of a point of order $k$ (namely in the sense of Young and Young and of Janiszewski) coincide. Thus we will use the term "in the classical sense" to name the concept and to distinguish it from the notion of order of a point used in the Menger-Urysohn theory of curves, where the

Received by the editors May 1, 1996.

1991 Mathematics Subject Classification. Primary 54C10, 54F15; Secondary 54F50.

Key words and phrases. Classical sense, confluent, continuum, dendroid, light, open mapping, order, smooth. 
concept is defined as, roughly speaking, the minimum cardinality of boundaries of small open neighborhoods of the point.

For the latter concept, G. T. Whyburn has proved (see [12, Corollary 7.31, p. 147]) that the Menger-Urysohn order of a point in a locally compact space is never increased under an open mapping. A natural question arises whether the same is true if order in the classical sense is under consideration. We give examples showing that it is not the case, but the property is kept even for a wider class of confluent mappings provided that the mapping is assumed to be light (i.e., it has totally disconnected point inverses) and that the domain space is a hereditarily unicoherent continuum. Examples are constructed showing that both assumptions are indispensable.

\section{Preliminaries}

All spaces considered in this paper are assumed to be Hausdorff. We denote by $\mathbb{N}$ the set of all positive integers, and by $\mathbb{R}$ the space of real numbers. Given two points $x$ and $y$ in either the plane $\mathbb{R}^{2}$ or the 3 -space $\mathbb{R}^{3}$, we denote by $x y$ the straight line segment joining $x$ with $y$. We shall use the concepts of Ls $A_{n}$ and $\operatorname{Lim} A_{n}$ (the limit superior and the limit of a sequence of sets $A_{n}$ in a space $X$ ) as defined in the Kuratowski monograph [6, §29, I-VI, p. 335-340].

By a continuum we mean a compact connected space. A continuum $X$ is said to be irreducible (between points $p$ and $q$ ) provided that no proper subcontinuum of $X$ contains both $p$ and $q$. An arc means a homeomorphic image of the closed unit interval $[0,1]$ of reals. The union of three arcs emanating from a point $p$ is called a simple triod provided that the singleton $\{p\}$ is the intersection of any two of these arcs. A space is said to be arcwise connected provided that for every two points $p, q \in X$ there exists in $X$ an arc from $p$ to $q$. A continuum $X$ is defined to be hereditarily unicoherent if the intersection of every two subcontinua of $X$ is connected. An arcwise connected and hereditarily unicoherent metric continuum is called a dendroid. A dendroid $X$ is said to be smooth provided that there is a point $v \in X$ (called an initial point of $X$ ) such that for each sequence $\left\{a_{n}\right\}$ of points of $X$ which converges to a point $a \in X$ the sequence of the arcs $v a_{n}$ converges to the arc va.

Let $\mathfrak{m}$ be a cardinal number. By an $\mathfrak{m}$-od with the center $p$ we mean the union of $\mathfrak{m}$ continua, every two of which have $p$ as the only common point. More precisely, a space $X$ is called an $\mathfrak{m}$-od with the center $p$ provided that there is a family $\left\{C_{s}: s \in S\right\}$ such that

1) $\operatorname{card} S=\mathfrak{m}$

2) for each $s \in S$ the set $C_{s}$ is a continuum, and $p \in C_{s}$;

3) if $s, t \in S$ and $s \neq t$, then $C_{s} \cap C_{t}=\{p\}$.

Let a continuum $X$ and a point $p \in X$ be given. Then $p$ is said to be a point of order at least $\mathfrak{m}$ in the classical sense, writing $\operatorname{ord}(p, X) \geq \mathfrak{m}$, provided that $p$ is the center of an $\mathfrak{m}$-od contained in $X$. We define $\operatorname{ord}(p, X)$ as the minimum cardinality $\mathfrak{m}$ for which the inequality $\operatorname{ord}(p, X) \geq \mathfrak{m}$ holds (i.e., $\operatorname{ord}(p, X) \geq \mathfrak{m}$, and condition $\operatorname{ord}(p, X) \geq \mathfrak{n}$ does not hold for any $\mathfrak{n}>\mathfrak{m})$. So, we then say that $p$ is a point of order $\mathfrak{m}$ in the classical sense, and we write $\operatorname{ord}(p, X)=\mathfrak{m}$.

The concept of the order in the classical sense was investigated by various authors mainly for dendroids ([1], [8], [9], [10], [11]), and for these continua $X$ it also was defined as the cardinality of the set of arc-components of $X \backslash\{p\}$ for $p \in X$ (compare 
e.g. [9, p. 422]). For dendroids, in the definition of an m-od (and, indirectly, of the concept of order in the classical sense) the members $C_{s}$ of the considered family can be taken as either continua, or irreducible continua, or arcs, and all the three concepts obtained in this way coincide. For arbitrary continua however such an equivalence is not true in general, and some authors take just arcs as the continua $C_{s}$ (see e.g. [4]).

Another commonly used concept of order at a point is that in the sense of Menger-Urysohn. For a detailed discussion of its properties the reader is referred to $[7, \S 51$, p. 274-307]. Here we merely mention that a point $p \in X$ is said to be of order at most $\mathfrak{m}$ in the sense of Menger-Urysohn provided that there exists a local basis $\mathcal{B}(p)$ of $X$ at $p$ such that

$$
\operatorname{card}(\operatorname{cl} U \backslash U) \leq \mathfrak{m} \quad \text { for each } U \in \mathcal{B}(p) .
$$

Then the Menger-Urysohn order of $p$ in $X$ is defined as the minimum cardinal $\mathfrak{m}$ satisfying the above condition.

The observation below is a consequence of the definitions.

1.1. Observation. The order of a point $p$ in a continuum $X$ in the classical sense is less than or equal to the order of $p$ in $X$ in the sense of Menger-Urysohn.

On the other hand, Menger's $n$-arc theorem (see [7, §51, I, p. 277]) says that if a metric continuum $X$ is locally connected and if a point $p \in X$ is of order at least $n \in \mathbb{N}$ in the sense of Menger-Urysohn, then there exist $n \operatorname{arcs} p a_{1}, \ldots, p a_{n}$ in $X$ which are pairwise disjoint except for $p$. Thereby, using Observation 1.1, we have the following.

1.2. Statement. Let $X$ be a locally connected continuum and $p \in X$. If the order of $p$ in $X$ in the sense of Menger-Urysohn is finite, then the two concepts of the order coincide; if it is infinite, then the order in the classical sense is infinite, too.

1.3. Example. There exists a locally connected continuum $X$ such that for any point $p \in X$ the order of $p$ in the sense of Menger-Urysohn is $\mathfrak{c}$, while the order of $p$ in the classical sense is $\aleph_{0}$.

Proof. Let $B_{n} \subset[0,1]$ be the union of $2^{n}$ pairwise disjoint closed intervals of length $1 / 3^{n}$ each such that $C=\bigcap\left\{B_{n}: n \in\{0\} \cup \mathbb{N}\right\}$ is the Cantor ternary set, i.e.,

$$
\begin{gathered}
B_{0}=[0,1], \quad B_{1}=[0,1 / 3] \cup[2 / 3,1], \\
B_{2}=[0,1 / 9] \cup[2 / 9,1 / 3] \cup[2 / 3,7 / 9] \cup[8 / 9,1], \quad \text { etc. }
\end{gathered}
$$

Put

$$
X^{\prime}=[0,1] \times C \cup \bigcup\left\{\left\{m / 2^{n}\right\} \times B_{n}: m \in\left\{0,1, \ldots, 2^{n}\right\} \text { and } n \in\{0\} \cup \mathbb{N}\right\} .
$$

This continuum contains points of order 2 (in any sense). Define $f: X^{\prime} \rightarrow X$ as a monotone mapping that shrinks every maximal free arc (i.e., an arc $a b$ such that $a b \backslash\{a, b\}$ is open in $\left.X^{\prime}\right)$ to a point. Then $X$ is a locally connected continuum such that the order of any point of $X$ in the sense of Menger-Urysohn equals $\mathfrak{c}$. To see that the order in the classical sense at any point $p \in X$ is $\aleph_{0}$ consider a family $\mathcal{C}$ of continua in $X$ pairwise disjoint out of $p$ and having $p$ as the only common point. Then the family $\left\{f^{-1}(C): C \in \mathcal{C}\right\}$ consists of subcontinua of $X^{\prime}$ having $f^{-1}(p)$ in common and disjoint out of $f^{-1}(p)$. Only four of them could be the unions of $f^{-1}(p)$ and some horizontal arcs in $X^{\prime}$. All others must contain some vertical 
arcs and, because there are only countably many vertical arcs, the family must be countable.

A mapping means a continuous function. A mapping $f: X \rightarrow Y$ between spaces $X$ and $Y$ is said to be:

- interior at a point $x \in X$, if for each open set $U$ about $x$ the image $f(x)$ is an interior point of $f(U)([12$, p. 149]);

- open, if $f$ maps each open set in $X$ onto an open set in $Y$ (note that $f$ is open if and only if it is interior at each point of $X$ );

- confluent, if for each subcontinuum $Q$ of $Y$ each component of $f^{-1}(Q)$ is mapped under $f$ onto $Q$ (note that each open mapping on a compact space is confluent [12, Chapter 8, (7.5), p. 148]);

- light, if each point-inverse has one-point components (note that if the pointinverses are compact, then this condition is equivalent to the property that they are zero-dimensional [12, p. 130]).

\section{TWO EXAMPLES}

G. T. Whyburn has proved (see [12, Corollary 7.31, p. 147]) that if a mapping $f$ from a locally compact space $X$ onto a space $Y$ is open (consequently $Y$ is locally compact, too; see $[12$, Note $1,(7.32)$, p. 279]), then the order of a point in the sense of Menger-Urysohn is never increased, that is, the order of $f(p)$ in $Y$ is less than or equal to the order of $p$ in $X$ in the sense of Menger-Urysohn. A question can be asked whether this is true if the order in the classical sense is considered. The answer is negative even if the domain and range spaces are dendroids. To show this we construct two examples. In both of them the key idea is that of a nonlight open mapping from the harmonic fan (i.e., the cone over $\{0\} \cup\{1 / n: n \in \mathbb{N}\}$ ) onto an arc, due to Michał Morayne, and described in [2, Example 7.1, p. 29] (compare also [2, Example 7.2, p. 30 and Example 11.17, p. 54]).

2.1. Example. There is a smooth dendroid $X$, a point $p \in X$ with $\operatorname{ord}(p, X)=$ 2 and an open mapping $f: X \rightarrow T$ from $X$ onto a simple triod $T$ such that $\operatorname{ord}(f(p), T)=3$.

Proof. In the Cartesian coordinates in $\mathbb{R}^{3}$ let

$$
v=(0,0,0), \quad p=(0,1,0), \quad q=(0,2,0), \quad a=(0,3,0), \quad b=(-1,2,0) .
$$

Let $\left\{t_{n} \in(1,2): n \in \mathbb{N}\right\}$ be a sequence such that $\operatorname{cl}\left\{t_{n} \in(1,2): n \in \mathbb{N}\right\}=[1,2]$. For each $n \in \mathbb{N}$ put

$$
a_{n}=(3 / n, 3,0), \quad b_{n}=(-1,2,3 / n), \quad c_{n}=\left(t_{n} / n, t_{n}, 0\right), \quad q_{n}=\left(t_{n} / n, 2,3 / n\right)
$$

and note that $c_{n} \in v a_{n}$.

Define

$$
X=v a \cup q b \cup \bigcup\left\{v a_{n} \cup c_{n} q_{n} \cup q_{n} b_{n}: n \in \mathbb{N}\right\}
$$

and note that

$$
v a=\operatorname{Lim} v a_{n}, \quad q b=\operatorname{Lim} q_{n} b_{n}, \quad p q=\operatorname{Ls}\left\{c_{n}\right\},
$$

and thus $p q=\operatorname{Ls} c_{n} q_{n}$, whence it follows that $X$ is a smooth dendroid having $v$ as its initial point. 
Denote by $T$ the limit triod of $X$, i.e., $T=q v \cup q a \cup q b$ and define the needed mapping $f: X \rightarrow T$ as follows:

$$
\begin{aligned}
& f(v)=v, \quad f(p)=q \quad \text { and } \quad f \mid v p: v p \rightarrow v q \text { is linear; } \\
& f \mid p q: p q \rightarrow\{q\} \quad \text { is constant; } \\
& f \mid(q a \cup q b): q a \cup q b \rightarrow q a \cup q b \quad \text { is the identity. }
\end{aligned}
$$

Thus $f \mid T: T \rightarrow T$ is defined. To define $f$ on $X \backslash T$, introduce an auxiliary notation. For each $n \in \mathbb{N}$ put

$$
\begin{gathered}
p_{n}=(1 / n, 1,0), \quad r_{n}=(2 / n, 2,0), \quad v_{n}=(0,2-1 /(n+1), 0), \\
u_{n}=(0,2+1 /(n+1), 0), \quad w_{n}=(-1 /(n+1), 2,0)
\end{gathered}
$$

and note that

$$
p_{n} \in v c_{n}, \quad r_{n} \in c_{n} a_{n}, \quad v_{n} \in p q \subset q v, \quad u_{n} \in q a, \quad w_{n} \in q b .
$$

Define

$$
f\left(p_{n}\right)=v_{n}, \quad f\left(c_{n}\right)=q, \quad f\left(r_{n}\right)=u_{n}, \quad f\left(a_{n}\right)=a, \quad f\left(q_{n}\right)=w_{n}, \quad f\left(b_{n}\right)=b,
$$

and take the following partial mappings as linear surjections:

$$
\begin{array}{ll}
f \mid v p_{n}: v p_{n} \rightarrow v v_{n}, & f \mid p_{n} c_{n}: p_{n} c_{n} \rightarrow v_{n} q, \\
f \mid r_{n} c_{n}: r_{n} c_{n} \rightarrow q u_{n}, & f \mid r_{n} a_{n}: r_{n} a_{n} \rightarrow u_{n} a, \\
f \mid c_{n} q_{n}: c_{n} q_{n} \rightarrow q w_{n}, & f \mid q_{n} b_{n}: q_{n} b_{n} \rightarrow w_{n} b .
\end{array}
$$

Continuity of $f$ follows from the definition. Since

$$
\operatorname{ord}(p, X)=2, \quad \operatorname{ord}(q, T)=3, \quad \text { and } \quad f(p)=q,
$$

we see that

$$
\operatorname{ord}(p, X)<\operatorname{ord}(f(p), f(X)) .
$$

We will show that for each point $x \in X$ the mapping $f$ is interior at $x$, whence openness of $f$ follows. If $x \in X \backslash p q$, then interiority of $f$ at $x$ is easy to verify. If $x \in p q$, let $U$ be any open set with $x \in U$. Then there is $n \in \mathbb{N}$ such that $c_{n} \in U$, and therefore $f(U)$ contains a simple triod which is the image under $f$ of the component of $U$ containing $c_{n}$. So, interiority of $f$ at $x$ follows, and the proof is complete.

2.2. Example. There is a plane dendroid $X$, an end point $p$ of $X$, and an open mapping $f: X \rightarrow A$ of $X$ onto an $\operatorname{arc} A$ such that $f(p)$ is an interior point of $A$.

Proof. In the plane $\mathbb{R}^{2}$ let

$$
v=(0,-1), \quad p=(0,1), \quad q=(0,0), \quad a=(1,0) .
$$

For each $n \in \mathbb{N}$ put

$$
\begin{gathered}
q_{n}=(1 / n,-1 / n), \quad a_{n}=(1+1 / n, 0), \quad b_{n}=(1 / n, 1 / n), \\
p_{n}=(0,1+1 / n), \quad d_{n}=(-1 / n,-1),
\end{gathered}
$$

and let

$$
A_{n}=v q_{n} \cup q_{n} a_{n} \cup a_{n} b_{n} \cup b_{n} p_{n} \cup p_{n} d_{n} .
$$


Define

$$
X=v p \cup q a \cup \bigcup\left\{A_{n}: n \in \mathbb{N}\right\}
$$

Thus

$$
v q=\operatorname{Lim} v q_{n}, \quad q a=\operatorname{Lim} q_{n} a_{n}=\operatorname{Lim} a_{n} b_{n}, \quad \text { and } \quad v p=\operatorname{Lim} p_{n} d_{n} .
$$

Therefore $X$ is a dendroid. Put $A=v q \cup q a$ and define the needed mapping $f: X \rightarrow A$, first on the limit triod $v p \cup q a$, as follows.

$$
f \mid A: A \rightarrow A \text { is the identity and } \quad f \mid p q: p q \rightarrow\{q\} \text { is constant. }
$$

To define $f$ out of this triod, for each $n \in \mathbb{N}$ consider points

$$
v_{n}=(0,-1 / n), \quad u_{n}=(1 / n, 0), \quad w_{n}=(2 / n, 0)
$$

and note that $v_{n} \in v q$ and $u_{n} \in q w_{n} \subset q a$. Further, let $\left\{t_{n} \in(0,1): n \in \mathbb{N}\right\}$ be a sequence such that $\operatorname{cl}\left\{t_{n} \in(0,1): n \in \mathbb{N}\right\}=[0,1]$, and let $\pi_{2}: \mathbb{R}^{2} \rightarrow \mathbb{R}$ be the second coordinate function, i.e., $\pi_{2}((x, y))=y$. For each $n \in \mathbb{N}$ let $e_{n}$ and $c_{n}$ be determined by the conditions

$$
\begin{array}{lll}
e_{n} \in p_{n} d_{n} & \text { and } & \pi_{2}\left(e_{n}\right)=0, \\
c_{n} \in p_{n} e_{n} & \text { and } & \pi_{2}\left(c_{n}\right)=t_{n} .
\end{array}
$$

Thus $p q=\operatorname{Ls}\left\{c_{n}\right\}$. Define

$$
\begin{array}{lll}
f\left(q_{n}\right)=q, & f\left(a_{n}\right)=a, & f\left(b_{n}\right)=w_{n}, \\
f\left(p_{n}\right)=u_{n}, & f\left(c_{n}\right)=q, & f\left(e_{n}\right)=v_{n}, \\
& f\left(d_{n}\right)=v, &
\end{array}
$$

and take the following partial mappings as linear surjections:

$$
\begin{array}{ll}
f \mid v q_{n}: v q_{n} \rightarrow v q, & f\left|q_{n} a_{n}: q_{n} a_{n} \rightarrow q a, \quad f\right| a_{n} b_{n}: a_{n} b_{n} \rightarrow a w_{n}, \\
f \mid b_{n} p_{n}: b_{n} p_{n} \rightarrow w_{n} u_{n}, & f\left|p_{n} c_{n}: p_{n} c_{n} \rightarrow u_{n} q, \quad f\right| c_{n} e_{n}: c_{n} e_{n} \rightarrow q v_{n}, \\
& f \mid e_{n} d_{n}: e_{n} d_{n} \rightarrow v_{n} v .
\end{array}
$$

Thus $f$ is well defined. Its continuity follows from the definition. Note that

$$
\operatorname{ord}(p, X)=1, \quad \operatorname{ord}(q, A)=2, \quad \text { and } \quad f(p)=q .
$$

As in the previous example we verify openness of $f$ by showing that for each $x \in X$ the mapping $f$ is interior at $x$. Note that for each $n \in \mathbb{N}$ the partial mapping $f \mid A_{n}: A_{n} \rightarrow A$ is open, thus $f$ is interior at each $x$ in $A_{n}$. If $x \in A$, then interiority of $f$ at $x$ is evident. So, it is enough to consider the case $x \in p q$. Let $U$ be any open set with $x \in U$. Then there is $c_{n} \in U$ for some $n \in \mathbb{N}$, and therefore $f(U)$ contains an open arc to which $f(x)=q$ belongs, and which is the image under $f$ of the component of $U$ containing $c_{n}$. So interiority of $f$ at $x$ follows, and the proof is complete.

Observe that the dendroid of Example 2.2 is not smooth. In connection with this we have a question.

2.3. Question. Let $f: X \rightarrow f(X)$ be an open mapping defined on a smooth dendroid $X$. Does then the inclusion

$$
f(E(X)) \subset E(f(X))
$$

hold true? 


\section{Confluent light MAPpings}

As the reader surely observed, neither of the mappings of Examples 2.1 and 2.2 is light: in both examples there is a point of the range space whose point-inverse is a nondegenerate continuum. It will be shown below that no such example of an open mapping $f$ increasing the order of a point in the classical sense does exist if $f$ has to be light. Moreover, an analog of the above mentioned Whyburn's theorem is true for a wider class of mappings, namely for confluent ones, provided that the domain space is a hereditarily unicoherent continuum (and that $f$ is assumed to be light).

3.1. Theorem. Let a continuum $X$ be hereditarily unicoherent. If a mapping $f: X \rightarrow f(X)$ is confluent and light, then for each point $p \in X$ we have

$$
\operatorname{ord}(f(p), f(X)) \leq \operatorname{ord}(p, X) .
$$

Proof. Let $\operatorname{ord}(f(p), f(X))=\mathfrak{m}$. Then there is a set $S$ with card $S=\mathfrak{m}$ and a family $\left\{C_{s}: s \in S\right\}$ of continua $C_{s} \subset f(X)$ such that

$$
s, t \in S \quad \text { and } \quad s \neq t \quad \text { implies } C_{s} \cap C_{t}=\{f(p)\} .
$$

Thus $\bigcup\left\{C_{s}: s \in S\right\}$ forms an m-od in $f(X)$ with the center $f(p)$. We will construct in $X$ an $\mathfrak{m}$-od with the center $p$. To this aim for each $s \in S$ let $A_{s}$ be the component of $f^{-1}\left(C_{s}\right)$ containing $p$. Take $s, t \in S$ with $s \neq t$ and observe that the intersection $A_{s} \cap A_{t}$ is a continuum by hereditary unicoherence of $X$. Further, $f\left(A_{s} \cap A_{t}\right) \subset$ $f\left(A_{s}\right) \cap f\left(A_{t}\right)=C_{s} \cap C_{t}=\{f(p)\}$ by confluence of $f$. Thus $p \in A_{s} \cap A_{t} \subset f^{-1}(f(p))$, and therefore $A_{s} \cap A_{t}=\{p\}$ by lightness of $f$. Hence $\bigcup\left\{A_{s}: s \in S\right\}$ is the required $\mathfrak{m}$-od. So, (3.2) is shown and the proof is finished.

3.3. Corollary. Confluent (in particular, open) light mappings of dendroids do not increase the order of points in the classical sense.

3.4. Remark. A mapping $f: X \rightarrow Y$ is said to be semi-confluent provided that for each continuum $Q \subset Y$ and for every two components $C_{1}$ and $C_{2}$ of $f^{-1}(Q)$ either $f\left(C_{1}\right) \subset f\left(C_{2}\right)$ or $f\left(C_{2}\right) \subset f\left(C_{1}\right)$. Obviously each confluent mapping is semiconfluent. Note that neither Theorem 3.1 nor Corollary 3.3 can be generalized to semi-confluent mappings. Namely $f:[-1,2] \rightarrow[0,2]$ defined by $f(x)=|x|$ is semiconfluent and light, and it maps an end point of the domain onto an interior point of the range.

It is seen by Examples 2.1 and 2.2 that lightness is an essential assumption in Theorem 3.1 and Corollary 3.3. We shall show that hereditary unicoherence is also essential in Theorem 3.1. The pseudo-arc (or any other hereditarily indecomposable continuum) will be used as a building block in the construction.

3.5. Example. There exist a continuum $X$, a point $p \in X$ and a confluent and light mapping $f: X \rightarrow Y$ such that

$$
\operatorname{ord}(p, X)=1 \quad \text { and } \quad \operatorname{ord}(f(p), Y)=2 .
$$

Proof. Let $\left\{P_{n}: n \in \mathbb{N}\right\}$ be a sequence of pseudo-arcs such that

$$
\begin{gathered}
\lim \operatorname{diam} P_{n}=0 ; \\
P_{n} \cap P_{n+1}=\left\{p_{n}\right\} ;
\end{gathered}
$$




$$
\begin{gathered}
P_{m} \cap P_{n}=\emptyset \text { for } m, n \in \mathbb{N} \text { and }|m-n|>1 ; \\
P_{n} \text { is irreducible between } p_{n-1} \text { and } p_{n} .
\end{gathered}
$$

Note that (3.6) and (3.7) imply that the sequence $\left\{p_{n}: n \in \mathbb{N}\right\}$ is convergent, and we denote

$$
p=\lim p_{n} .
$$

Let $\left\{P_{n}^{\prime}: n \in \mathbb{N}\right\}$ and $\left\{p_{n}^{\prime}: n \in\{0\} \cup \mathbb{N}\right\}$ be other sequences of pseudo-arcs and of points, respectively, satisfying analogous conditions to (3.6)-(3.9). Assume moreover, that $p_{2 n}=p_{2 n}^{\prime}$ for $n \in\{0\} \cup \mathbb{N}$ and that

$$
\left(\bigcup\left\{P_{n}: n \in \mathbb{N}\right\}\right) \cup\left(\bigcup\left\{P_{n}^{\prime}: n \in \mathbb{N}\right\}\right)=\left\{p_{2 n}: n \in\{0\} \cup \mathbb{N}\right\},
$$

i.e., $P_{m} \cap P_{n}^{\prime}=\emptyset$ for $m, n \in \mathbb{N}$ with $|m-n|>1$, or $m=2 k+1$ and $n=2 k+2$, or $m=2 k+2$ and $n=2 k+1$, and that any two of the four continua $P_{2 n}, P_{2 n}^{\prime}, P_{2 n+1}$, $P_{2 n+1}^{\prime}$ have the point $p_{2 n}$ in common only.

Put

$$
X=\{p\} \cup \bigcup\left\{P_{n} \cup P_{n}^{\prime}: n \in \mathbb{N}\right\} .
$$

Let $Q$ and $Q^{\prime}$ be pseudo-arcs such that $Q \cap Q^{\prime}=\{q\}$. Define

$$
Y=Q \cup Q^{\prime},
$$

and let $f: X \rightarrow Y$ be a mapping satisfying the following conditions:

$$
\begin{gathered}
f\left(p_{2 n}\right)=q \text { for } n \in\{0\} \cup \mathbb{N} ; \\
f\left(P_{1}\right)=Q \text { and } f\left(P_{1}^{\prime}\right)=Q^{\prime} \\
f\left(P_{n}\right) \subset Q \text { and } f\left(P_{n}^{\prime}\right) \subset Q^{\prime} \\
f \mid P_{n} \text { and } f \mid P_{n}^{\prime} \text { are embeddings. }
\end{gathered}
$$

We will show that $f$ is light and confluent. Really, $f^{-1}(q)=\left\{p_{2 n}: n \in\{0\} \cup \mathbb{N}\right\}$, so it is countable, and for any other point $y$ in $Y$ the set $f^{-1}(y)$ is finite. Thus $f$ is light.

To see that it is confluent take any continuum $B \subset Y$ and consider three cases.

Case 1. $B \subset Q$. Then

$$
f^{-1}(B) \subset\{p\} \cup \bigcup\left\{P_{n}: n \in \mathbb{N}\right\} .
$$

Note that the partial mapping $g=f \mid\left(\{p\} \cup \bigcup\left\{P_{n}: n \in \mathbb{N}\right\}\right)$ is a mapping onto the pseudo-arc $Q$; thus it is confluent ([3, Theorem 4, p. 243]). Since components of $f^{-1}(B)$ coincide with ones of $g^{-1}(B)$, the conclusion follows.

Case 2. $B \subset Q^{\prime}$. This is quite the same as Case 1 .

Case 3. $q \in B$. Then $B=C \cup C^{\prime}$, where $C=B \cap Q$ and $C^{\prime}=B \cap Q^{\prime}$. Let $K$ be a component of $f^{-1}(B)$. If $B \neq\{q\}$, then $p_{2 n} \in K$ for some $n \in \mathbb{N}$. By Cases 1 and 2 there are continua $L$ and $L^{\prime}$ in $K$ such that

$$
p_{2 n} \in L \cap L^{\prime}, \quad f(L)=C \text { and } f\left(L^{\prime}\right)=C^{\prime} .
$$

Thus $f(K) \supset f(L) \cup f\left(L^{\prime}\right)=C \cup C^{\prime}=B$. The proof is finished. 


\section{ACKNOWLEDGEMENT}

The authors thank Sergio Macias who posed the problem discussed in the paper and encouraged them to work on the subject.

\section{REFERENCES}

1. J. J. Charatonik, On ramification points in the classical sense, Fund. Math. 51 (1962), 229252. MR 26:743

2. J. J. Charatonik, W. J. Charatonik and S. Miklos, Confluent mappings of fans, Dissertationes Math. (Rozprawy Mat.) 301 (1990), 1-86. MR 91h:54056

3. H. Cook, Continua which admit only the identity mapping onto non-degenerate subcontinua, Fund. Math. 60 (1967), 241-249. MR 36:3315

4. C. A. Eberhart, J. B. Fugate and G. R. Gordh, Jr., Branchpoint covering theorems for confluent and weakly confluent maps, Proc. Amer. Math. Soc. 55 (1976), 409-415. MR 53:14450

5. Z. Janiszewski, Sur les continus irréductibles entre deux points, Journal de l'Ecole Polytechnique (2) 16 (1912), 79-170.

6. K. Kuratowski, Topology, vol. 1, Academic Press and PWN, 1966. MR 36:840

7. K. Kuratowski, Topology, vol. 2, Academic Press and PWN, 1968. MR 41:4467

8. A. Lelek, On plane dendroids and their end points in the classical sense, Fund. Math. 49 (1960/61), 301-319. MR 24:A3631

9. J. Nikiel, A characterization of dendroids with uncountably many end points in the classical sense, Houston J. Math. 9 (1983), 421-432. MR 85e:54041

10. J. Nikiel, On dendroids and their ramification points in the classical sense, Fund. Math. 123 (1984), 39-46. MR 86c:54034

11. J. Nikiel, On dendroids and their end points and ramification points in the classical sense, Fund. Math. 124 (1984), 99-108. MR 86g:54051

12. G. T. Whyburn, Analytic topology, reprinted with corrections 1971, Amer. Math. Soc. Colloq. Publ. 28, Providence, 1942. MR 32:425

13. W. H. Young and G. Ch. Young, The theory of sets of points, Cambridge University Press, Cambridge, 1906. MR 57:16079

Mathematical Institute, University of WrocŁaW, Pl. Grunwaldzki 2/4, 50-384 WrocŁAW, POLAND

E-mail address: jjc@math.uni.wroc.pl

Current address: Instituto de Matemáticas, UNAM, Circuito Exterior, Ciudad Universitaria, 04510 México, D. F., México

E-mail address: jjc@gauss.matem.unam.mx

Departamento de Matemáticas, Facultad de Ciencias, Unam, Circuito Exterior, Ciudad Universitaria, 04510 México, D. F., MÉxico

E-mail address: wjcharat@math.uni.wroc.pl

E-mail address: wjcharat@lya.fciencias.unam.mx 